OPEN ACCESS

Edited by:

Andrew Burgess,

Anzac Research Institute, Australia

Reviewed by:

David Q. Matus,

Stony Brook University, United States

Bojana Gligorijevic,

Temple University, United States

Hava Gil-Henn

Bar-Ilan University, Israel

${ }^{*}$ Correspondence:

Evelyn Lattmann

evelyn.lattmann@im/s.uzh.ch

Specialty section:

This article was submitted to

Cell Growth and Division,

a section of the journal

Frontiers in Cell and Developmental

Biology

Received: 10 October 2020 Accepted: 09 December 2020

Published: 06 January 2021

Citation:

Lattmann E, Deng $T$ and Hajnal $A$

(2021)

To Divide or Invade: A Look Behind the

Scenes of the Proliferation-Invasion Interplay in the Caenorhabditis elegans Anchor Cell. Front. Cell Dev. Biol. 8:616051. doi: 10.3389/fcell.2020.616051

\section{To Divide or Invade: A Look Behind the Scenes of the Proliferation-Invasion Interplay in the Caenorhabditis elegans Anchor Cell}

\author{
Evelyn Lattmann ${ }^{1 *}$, Ting Deng ${ }^{1,2}$ and Alex Hajnal ${ }^{1}$ \\ ${ }^{1}$ Department of Molecular Life Sciences, University of Zurich, Zurich, Switzerland, ${ }^{2}$ Molecular Life Science PhD Program, \\ University and ETH Zurich, Zurich, Switzerland
}

Cell invasion is defined by the capability of cells to migrate across compartment boundaries established by basement membranes (BMs). The development of complex organs involves regulated cell growth and regrouping of different cell types, which are enabled by controlled cell proliferation and cell invasion. Moreover, when a malignant tumor takes control over the body, cancer cells evolve to become invasive, allowing them to spread to distant sites and form metastases. At the core of the switch between proliferation and invasion are changes in cellular morphology driven by remodeling of the cytoskeleton. Proliferative cells utilize their actomyosin network to assemble a contractile ring during cytokinesis, while invasive cells form actin-rich protrusions, called invadopodia that allow them to breach the BMs. Studies of developmental cell invasion as well as of malignant tumors revealed that cell invasion and proliferation are two mutually exclusive states. In particular, anchor cell (AC) invasion during Caenorhabditis elegans larval development is an excellent model to study the transition from cell proliferation to cell invasion under physiological conditions. This mini-review discusses recent insights from the $C$. elegans AC invasion model into how $\mathrm{G} 1$ cell-cycle arrest is coordinated with the activation of the signaling networks required for BM breaching. Many regulators of the proliferation-invasion network are conserved between $C$. elegans and mammals. Therefore, the worm may provide important clues to better understand cell invasion and metastasis formation in humans.

\section{Keywords: anchor cell, invasion, proliferation, EGL-43, EVI1, cell cycle, basement membrane}

\section{INTRODUCTION}

AC invasion in Caenorhabditis elegans is an excellent model to investigate the various checkpoints regulating developmental cell invasion, including G1 cell cycle arrest required for BM breaching (Matus et al., 2015; Deng et al., 2020; Medwig-Kinney et al., 2020). AC invasion occurs during the mid- to late-L3 larval stage in order to establish a connection between the uterus and developing vulva (Sherwood and Sternberg, 2003) (Figure 1). The importance of the morphogenetic events triggered by AC invasion manifests in mutants with defective AC invasion. For example, loss-of-function mutations in the AP-1 transcription factor fos-1 (FOS, FOSL1, FOSL2), the gene encoding a key invasion driver, lead to a protruding vulva (Pvl) phenotype and 


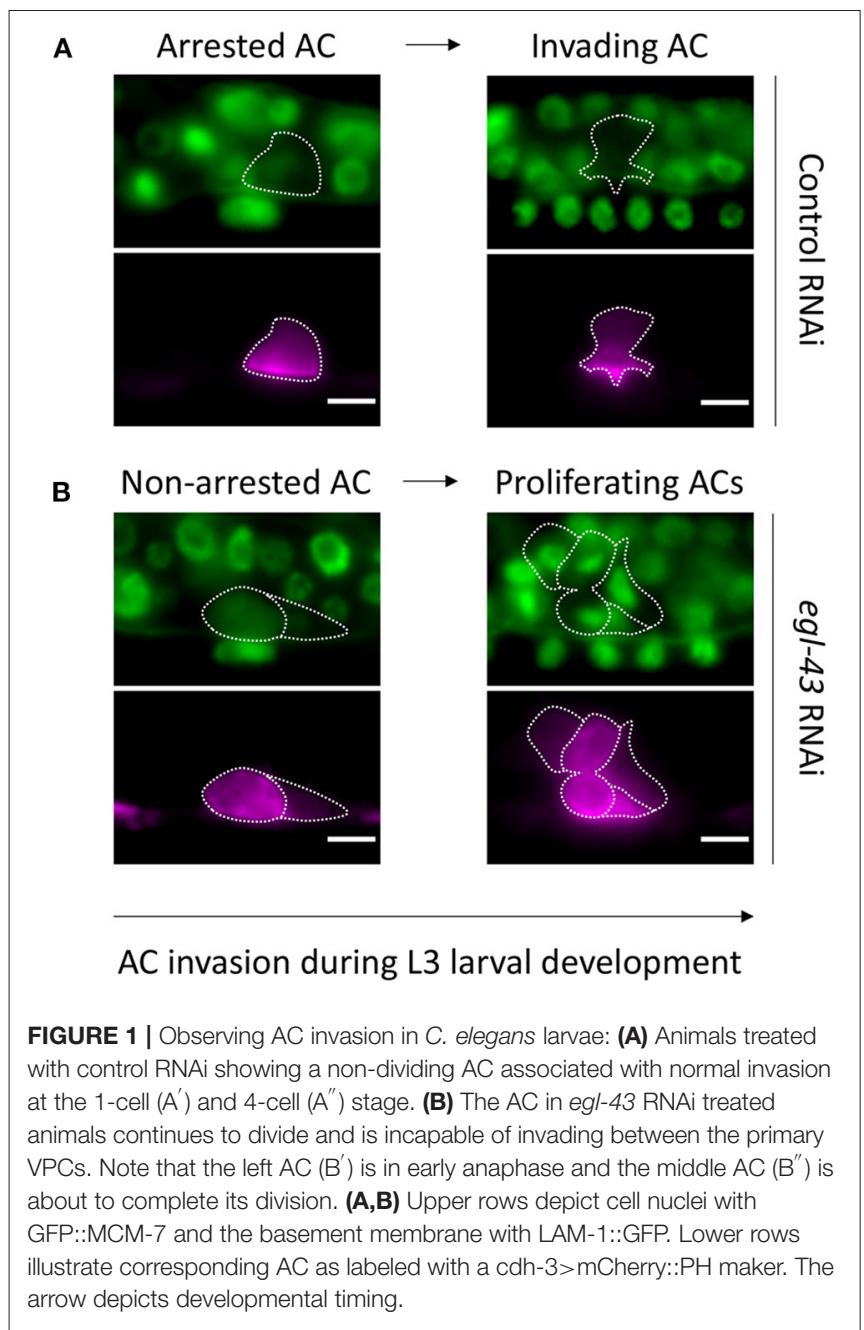

adult sterility (Sherwood et al., 2005). The AC is derived from one of two primordial gonadal cells, Z1 and Z4 (Kimble and Hirsh, 1979). Two of the $12 \mathrm{Z1}$ and Z4 descendant Z1.ppp and Z4.aaa, have an equal potential to adopt the default AC fate, but only one cell becomes the AC during the L2 larval stage, while the other one is acquiring the ventral uterine (VU) fate stage (Kimble and Hirsh, 1979; Kimble, 1981). A positive feedback loop established by upregulation of the Notch ligand LAG-2 (DSL) in the future AC and by lateral inhibition via activation of the Notch receptor LIN-12 (Notch) in the adjacent VU precursor underly the AC/VU decision (Seydoux and Greenwald, 1989; Greenwald and Kovall, 2013). hlh-2 (TCF3, TCF4,TCF12) encodes a basic helix-loop-helix transcription factor that upregulates lin-12 expression in the presumptive VU cell (Attner et al., 2019). The initial imbalance in Notch signaling is driven by the onset of $h l h-2$ expression, which is linked to the relative birth order of the AC/VU ancestor cells Z1.ppp and Z4.aaa. Later on, HLH-2 is post-transcriptionally silenced in the future VU cell, whereas HLH-2 in the AC binds to the E-boxes in the lag2 promoter, thus maintaining LAG-2 expression and establishing the positive feedback loop (Karp and Greenwald, 2004). In vitro assays have suggested that HLH-2 also binds to E-boxes in the promoter of egl-43, which encodes a zinc finger transcription factor homologous to the human EVI1 proto-oncogene and contributes to the VU/AC decision (Hwang et al., 2007; Rimann and Hajnal, 2007). While egl-43 is important for G1 arrest and pro-invasive gene expression in the AC (see below), egl-43 is also expressed in the proliferating VU cells where it promotes VU fate specification (Rimann and Hajnal, 2007). Moreover, HLH-2 is required in the $\mathrm{AC}$ to upregulate expression of the epidermal growth factor homolog LIN-3 (EGF), which acts as inductive signal during vulval cell fate specification (Hwang and Sternberg, 2004; Sternberg, 2005).

\section{REGULATION OF AC INVASION}

While egl-43 and $h l h-2$ are important for the AC/VU fate decisions, they later play a central role in inducing the invasive AC fate by enabling G1 cell cycle arrest and activating the expression of pro-invasive genes, which are controlled by the C. elegans ortholog of human FOS fos-1 (Sherwood et al., 2005) (Figure 2). Among the fos-1 target genes are several conserved extracellular matrix genes, such as hemicentin him4 (HMCN1), the zinc metalloproteinases genes (MMPs: $z m p$ 1, zmp-3, and $z m p-6)$, the papilin homolog mig-6 (PAPLN), or the protocadherin $c d h-3(P D C H)$, as well as actin cytoskeleton regulators such the small GTPases mig-2 (RHOG) and ced-10 $(R A C)$ or the lamellipodin homolog mig-10 (RAPH1) (Sherwood et al., 2005; Ihara et al., 2011; Wang et al., 2014a; Matus et al., 2015). AP-1 also drives tumor invasion in several types of human cancer (Ozanne et al., 2006). Furthermore, there exists some degree of conservation at the level of target genes, such as MMP1, MMP3, and MMP9 that have been shown to be under direct AP1 control in a variety of cellular contexts (Angel et al., 1987; Lee et al., 1987; Benbow and Brinckerhoff, 1997).

G1 cell cycle arrest of the $\mathrm{AC}$ is a pre-requisite for proinvasive gene expression and formation of invasive protrusions, as S-phase or G2 entry of the AC prevents invasion (Matus et al., 2015). Moreover, the activity of HDA-1, a component of the nucleosome remodeling deacetylase (NuRD) complex among others, is important for linking the G1 arrest to fos-1-mediated gene expression and formation of invadopodia-like protrusions (Matus et al., 2010). Besides HDA-1, MEP-1 and the cytosolic chaperonin containing TCP-1 (CCT) complex act upstream of fos- 1 and actin polarity pathways. Interestingly, in the germ cells MEP-1 interacts with the NuRD complex to maintain the somatic differentiation (Unhavaithaya et al., 2002), and also the CCT complex interacts with HDAC1 (Dekker et al., 2008; Banks et al., 2018). However, how these chromatin modifiers precisely act in the invading AC remains to be studied.

A positive regulation of fos- 1 by egl-43 has recently been observed (Deng et al., 2020; Medwig-Kinney et al., 2020). Thus, egl-43 plays a dual role in AC invasion, on the one hand by inducing G1 cell cycle arrest and on the other hand by activating expression of fos-1. Despite acting downstream of the G1 arrest and egl-43, fos-1 positively regulates $h l h-2$ and egl-43 expression in the AC, revealing a complex regulatory 


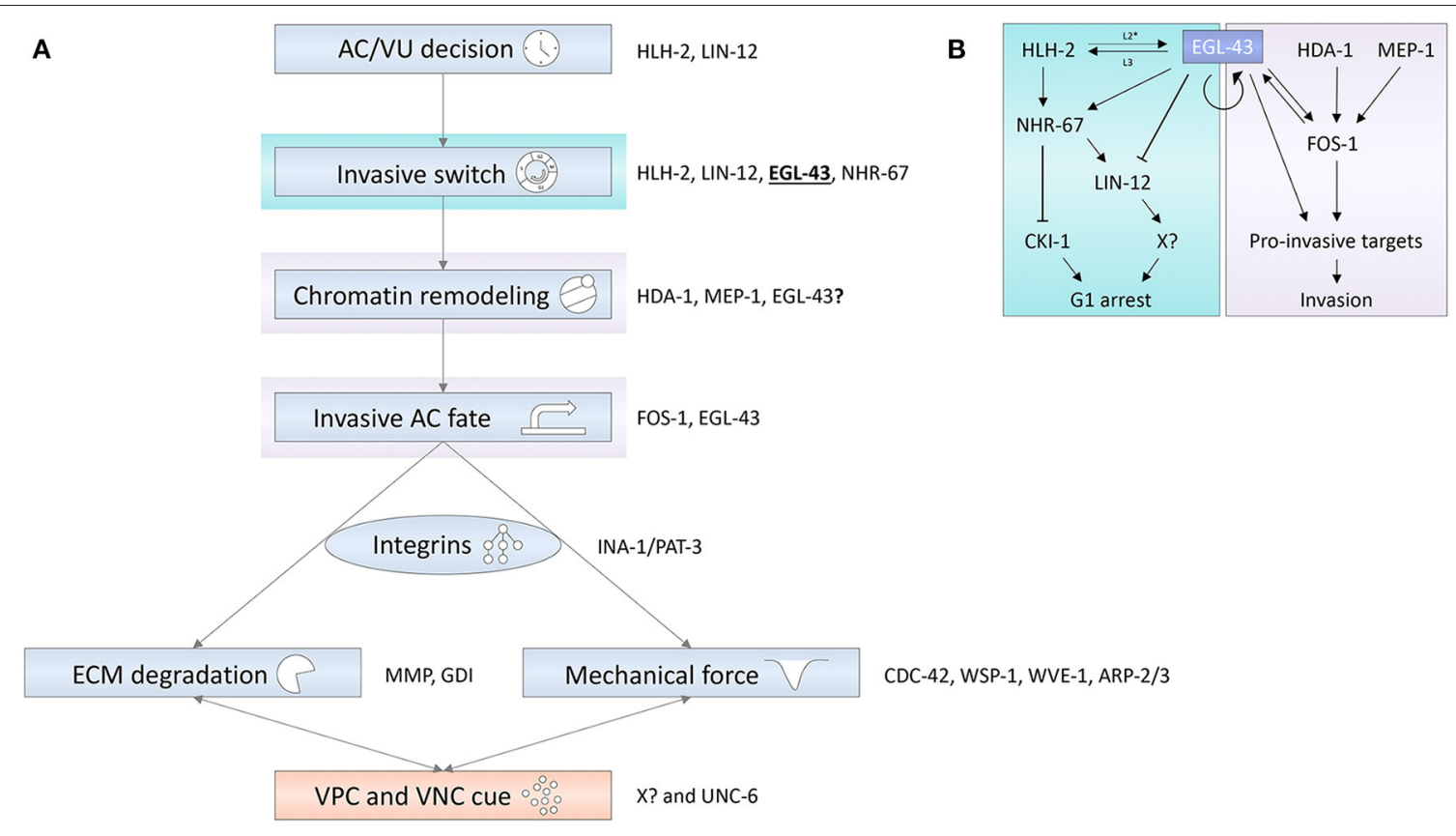

FIGURE 2 | (A) Different regulatory layers are integrated to control AC invasion. Blue shaded boxes depict cell-autonomous processes in the AC; the red shaded box represents paracrine signaling processes mediated by the VPCs and the ventral nerve cord (VNC). Some of the genes encoding factors important for the depicted processes are shown on the right of the rectangles. (B) The EVI1 homolog EGL-43 is a central node in a transcription factor network coordinating the different signaling layers that induce $\mathrm{G} 1$ cell cycle arrest and promote pro-invasive gene expression. Arrows refer to activating and T-bars to inhibitory interactions.

network formed by these three transcription factors (MedwigKinney et al., 2020). While fos-1 loss-of-function mutants exhibit fully penetrant BM breaching defects, mutations in the FOS-1 target genes do not cause strong AC invasion defects (Sherwood et al., 2005). Even in cdh-3, him-4, zmp-1 triple mutants, the AC failed to invade in only $25 \%$ of the animals (Kelley et al., 2019). These findings have suggested the existence several partially redundant invasion pathways that ensure robust BM breaching. A study in human ovarian carcinoma cells has found several overlapping targets of the AP-1 and EVI1 transcription factors (Bard-Chapeau et al., 2012). Therefore, fos- 1 targets may be co-regulated in a cooperative fashion by egl-43. mig-10 is one example for an antagonistic regulation, whereby fos- 1 activates and egl-43 inhibits mig-10 expression (Wang L. et al., 2014; Wang et al., 2014a).

Apart from the enzymatic dissolution of the $\mathrm{BM}$, breaching and invasion also require physical forces generated by invasive protrusions. These forces are exerted by actin-rich protrusions that depend on the actin nucleator complex Arp2/3, which is activated in the AC predominantly by WSP- $1 / \mathrm{N}-\mathrm{WASP}$ and to a lesser extent by WVE-1/WAVE (Cáceres et al., 2018). Upstream of WSP-1/N-WASP acts the GTPase CDC-42 (Lohmer et al., 2016). However, MIG-2/Rac signaling can compensate for a loss of WSP-1/N-WASP activation when CDC- 42 is absent (Cáceres et al., 2018). Moreover, in the absence of the entire CDC-42/Cdc42-MIG-2/Rac-WSP-1/N-WASP pathway, WVE-1 signaling can be activated by CED-10/Rac signaling (Lohmer et al., 2016; Cáceres et al., 2018). Activation of CDC-42 in the AC depends on one or several unknown diffusible cues secreted by the induced primary vulval precursor cells, together with an UNC-6 (NTN1) Netrin signal released from the ventral nerve cord (Ziel et al., 2009; Lohmer et al., 2016). These two signals guide the AC protrusions in order to breach the $\mathrm{BM}$ and to invade precisely at the vulval midline between the primary VPCs (Sherwood and Sternberg, 2003). The UNC-6 Netrin signal directs the AC protrusions toward the ventral midline by binding to the UNC40 (DCC/NEO1) receptor that is polarized toward the invasive membrane in the AC (Ziel et al., 2009). The UNC-6/UNC40 netrin pathway is required for the polarized enrichment of actin regulators, such as phospholipid phosphatidylinositol 4,5-bisphosphate (PI(4,5)P2), MIG-2 (RHOG), CED-10 (RAC), UNC-115 (ABLIM1), and UNC-34 (EVL). Finally, UNC40-directed lysosomal exocytosis, which delivers MMPs and membrane fractions to the invadopodia-like protrusions, leads to the formation of a single AC protrusion (Naegeli et al., 2017). However, unc-6 and unc-40 mutants do not exhibit identical phenotypes, since UNC-40 can function in a ligand-independent way to regulate F-actin polarity and partially compensate for the lack of the UNC-6 ligand (Wang et al., 2014b). Both unc40 and unc- 6 mutants display a delayed invasion, but they do not block invasion (Ziel et al., 2009), indicating that the Netrin signal and the cue from the vulval cells are partially redundant. Thus, the activation of the CDC- 42 pathway and the force generation by the invasive protrusions depend on multiple cell non-autonomous cues.

In addition to the CDC-42 pathway, the vulval cue regulates the Rab GDP dissociation inhibitor (GDI), which controls membrane trafficking to form plasma membrane protrusion 
(Lohmer et al., 2016). The molecular nature of the signals activating the CDC-42 and GDI pathways in the AC remains an enigma. G-Protein coupled receptors (GPCR), receptor tyrosine kinases (RTKs) and integrins are known to be involved in CDC42 activation. However, besides the Netrin cue, no other secreted signal or receptor required for $\mathrm{AC}$ invasion has been found to date.

Signaling through the INA-1 (ITGA3, ITGA6, ITGA7)/PAT-3 (ITGB1) alpha/beta integrin complex acts upstream of the Netrin pathway to control the recruitment of F-actin to the AC plasma membrane (Hagedorn et al., 2009). The different phenotypes of ina-1 and pat-3 mutants compared to Netrin pathway mutants suggest that the integrins play a broader role in recruiting UNC40 and F-actin to the plasma membrane, while the netrin signal provides the directional information for a specific recruitment to the invasive membrane front.

\section{THE EVI1 HOMOLOG egl-43 COORDINATES AC PROLIFERATION AND INVASION}

As discussed above, egl-43 is necessary for G1 cell cycle arrest of the AC. In addition, the nuclear hormone receptor gene nhr-67 (NR2E1) is critical for G1 cell cycle block in the AC (Matus et al., 2015). Removal of either of these two transcription factors results in AC proliferation, reduced pro-invasive gene expression, impaired invasive membrane organization and lack of invadopodia-like protrusion formation. This implies that egl43 and $n h r-67$ are both essential for halting cell cycle progression in the invasive AC. How these transcription factors interact and coordinate the cell cycle arrest with invasion has been the subject of two recent publications.

Medwig-Kinney et al. (2020) and Deng et al. (2020) studied the relationship between $h l h-2$, egl-43, fos-1, and nhr-67 during cell invasion and identified a network defining a cell-cycle dependent axis of invasion control, whereby egl-43 regulates $n \mathrm{hr}$ 67 expression in an hlh-2-dependent and -independent manner (Figure 2). NHR-67 then establishes the G1 arrest in the AC by activating expression of the CDK inhibitor CKI-1 (Matus et al., 2015). Moreover, egl-43 positively regulates fos-1 expression, indicating an additional, cell-cycle independent role of egl-43 in controlling AC invasion. In this model, egl-43 emerges as a central player linking G1 arrest to the cell-cycle independent invasion network by activating fos- 1 and $n h r-67$ expression and forming several positive feedback loops including autoregulation. CHIP-seq data indicated that EGL-43 may act by directly binding to enhancer elements in the fos- 1 locus (Deng et al., 2020).

However, egl-43 and $n h r-67$ may also act in distinct pathways, since $n h r-67$ establishes the cell cycle arrest in the AC primarily via CKI-1 expression, whereas egl-43 appears to restrict AC proliferation predominantly by inhibiting the LIN-12 Notch pathway. Surprisingly, the ectopic activation of LIN-12 Notch signaling in the already differentiated AC was sufficient to induce proliferation. On the other hand, inhibiting lin-12 notch expression efficiently suppressed the AC proliferation caused by loss of egl-43, but not nhr-67 function (Deng et al., 2020). In several cell types, Notch signaling directly promotes G1-S transition. For example, Notch regulates cyclin D1 expression in mammalian kidney, breast epithelial cells and cardiomyocytes (Ronchini and Capobianco, 2001; Campa et al., 2008; Cohen et al., 2010), activates dE2F1 and cyclin A expression in the Drosophila photoreceptor precursors (Baonza and Freeman, 2005) and negatively regulates the CDK inhibitors $\mathrm{p} 27^{\mathrm{Kip} 1}$ and p2 ${ }^{\mathrm{Cip} 1}$ to promote S-phase entry (Noseda et al., 2005; Sarmento et al., 2005).

Taken together, two distinct mechanisms ensure G1 arrest of the AC; EGL-43 inhibits S-phase entry by repressing Notch signaling, while NHR-67 maintains the G1 arrest of the AC by activating CKI-1 expression. This double authentication system established by NHR-67 and EGL-43-mediated cell cycle inhibition may add the developmental robustness necessary for the AC to adopt a stable invasive fate. Further studies will be needed to probe this hypothesis and identify additional cell cycle regulators controlled by $n h r-67$, egl-43 and the lin-12 Notch pathway.

\section{Context-Dependent Regulation of Proliferation by egl-43 and lin-12 Notch Signaling}

egl-43 is not only expressed in the AC but also in the VU cells that undergo three rounds of cell division. This raises the important question of cell context-dependent specificity. In fact, the absence of terminal differentiation ( $\pi$-fate) markers in the VU cells after inhibition of egl-43 might even hint at a defect in VU cell proliferation. In the VU cells egl-43 seems to be positively regulated by Notch signaling, which turns around the relationship observed in the AC. Whether this difference in LIN12 activity is caused by a different type of regulation remains to be examined. DNA binding of the human EGL-43 homolog EVI1 is modulated by serine phosphorylation through casein kinase II (CK2) and PP1A (Bard-Chapeau et al., 2013). In particular these phosphorylation sites have been shown to modulate EVI1 DNA binding to ETS-like binding motifs (Bard-Chapeau et al., 2013), often present in cell cycle regulator genes (Bard-Chapeau et al., 2012). CK2 also phosphorylates the intracellular domain of NOTCH (NICD), which results in differential binding to the Notch transcription factor complex [LAG-1 (CBF1/RBP-J)/SEL8 (MAML)] and changes the pattern of NOTCH driven target gene expression (Ranganathan et al., 2011). Hence, differential phosphorylation of EVI1 and NOTCH may be important for their context-dependent activities. Alterations in EVI1 regulation have also been attributed to the differential expression of the long vs. the short isoform. In the C. elegans AC, the long isoform EGL$43 \mathrm{~L}$ is the dominant factor regulating G1 arrest and pro-invasive gene expression, for which the short isoform is dispensable (Deng et al., 2020; Medwig-Kinney et al., 2020). One possibility is therefore that the short EGL-43S isoform plays the opposite role in promoting VU cell proliferation.

\section{Timing of the G1 Arrest}

Besides the spatial context established through cell-cell signaling, the timing of the G1 arrest in the AC needs consideration. Studies with heterochronic mutants reveal that the timing of invasion is intrinsically programmed in the AC. For example, in a lin-28 (LIN28A, LIN28B) mutant, where the primary 
VPC is precociously induced, the AC invades at the normal developmental time in mid-L3, but the vulva is already at the morphogenetic stage (L4 stage) (Sherwood and Sternberg, 2003). Thus, the competence to respond to extracellular invasion cues likely depends on a cell-autonomous molecular clock in the AC.

While hlh-2, nhr-67 and egl-43 are required for the maintenance of the G1 arrest throughout the L3 phase, the G1 arrest is already established at the L2 stage, shortly after the specification of the AC. Low fos-1 expression can be seen in the newly specified AC and expression levels gradually increase until invasion begins (Sherwood and Sternberg, 2003; Sherwood et al., 2005; Medwig-Kinney et al., 2020). Since HLH-2, EGL-43, and NHR-67 are already expressed during the AC/VU decision, it seems likely that the induction of the G1 arrest is tied to AC fate specification at the early L2 larval stage. In this context it is important to note that the early L2 function of egl-43 is required for AC invasion during the later L3 stage, which was shown by early expression of the dominant-negative egl-43 PR domain in the AC (Hwang et al., 2007). An invasion defect was only observed when the PR domain was expressed from an egl-43 promoter with functional E-boxes, required for early expression of egl-43 at the mid L2 stage (Hwang and Sternberg, 2004; Hwang et al., 2007). Since egl-43 expression in the newly formed AC may depend on hlh-2 activity (Hwang et al., 2007), the onset of $h l h-2$ expression could serve as a molecular clock to set the time of invasion. However, no clear timing dependency has been observed in a recent study examining at an egl-43 reporter upon hlh-2 RNAi at the L3 stage (Medwig-Kinney et al., 2020). Since the specification of the AC itself depends on $h l h-2$, early expression changes in the newly formed AC cannot be addressed by this approach. Different combinations of bHLH transcription factors represent distinct codes for cell fate specification (Sallee et al., 2017). Thus, hlh-2 might integrate developmental timing with spatial cues to program the AC for the G1-arrested invasive state.

\section{A Pro-invasive Chromatin Landscape?}

Down-regulation of $h d a-1$ suggested a role for the NuRD complex and histone-deacetylation in AC invasion. Additional chromatin modifiers involved, such as components of the MEC complex, remain to be identified. It will be of interest to investigate how egl-43 and $n h r-67$ interact with the known (i.e., HDA-1 and MEP-1) and the yet to be identified epigenetic regulators of AC invasion. A potential function of egl-43 in epigenetic gene regulation is suggested by the interactions of human EVI1 with different chromatin modifier complexes (BardChapeau et al., 2013). For example EVI1 recruits the corepressor $\mathrm{CrBP}$ to the SMAD3 promoter to repress $\mathrm{TGFb}$ signaling (Izutsu et al., 2001), interacts with components of the SWI/SNF to derepress E2F1 expression and binds to the polycomb complex to inhibit PTEN signaling (Chi et al., 2003; Yoshimi et al., 2011). Isoform-specific interactions of EVI1 have been observed with components of the NuRD complex (Ivanochko et al., 2019), suggesting that context dependency may also affect the interaction with epigenetic regulators. Thus, an analysis of EGL43 protein interaction partners may reveal epigenetic regulators that are critical for establishing the invasive AC fate.

\section{Can the Dichotomy Between Proliferation and Invasion Be Applied to Cancer Cells?}

A similar dichotomy between proliferation and invasion has been observed in human cancer cells. The "go or grow" concept states that cells must choose one of three options; (1) to proliferate, (2) to migrate, or to (3) terminally differentiate (Hatzikirou et al., 2012). In this context, the colony-stimulating factor1 receptor CSF1R and the non-receptor tyrosine kinase and Arg/Abl2 are important players in regulating the invasionproliferation switch in cancer (Gil-Henn et al., 2013; Patsialou et al., 2015). This dichotomy also manifests in many cancer cells that undergo EMT-like changes, which coincide with their proliferation arrest (Kohrman and Matus, 2017). It has also been proposed that cancer cells, especially in human melanoma, switch back and forth between an epithelial, proliferative and a mesenchymal, invasive state (Hoek et al., 2008) However, the direct observation of invading melanoma cells in real time has again challenged this model (Haass et al., 2014). In C. elegans, AC-specific expression of the p21 homolog CKI-1 restored the invasive fate even when the $\mathrm{AC}$ was induced to proliferate, suggesting a plasticity between the proliferative and invasive states. Similar to the situation in C. elegans, a switch between invasion and proliferation has been proposed for breast cancer based on the finding that loss of G1 phase inhibitor p21 (CKI) or overexpression of cyclin $\mathrm{E}$ lead to suppression of metastasis (Qian et al., 2013). Furthermore, a link between G1 cell cycle and invadopodia formation during breast carcinoma invasion has recently been reported (Bayarmagnai et al., 2019). Though, the fact that invadopodia precursors can be assembled throughout the cell cycle leaves a more nuanced picture of the proliferationinvasion switch (Bayarmagnai et al., 2019).

\section{CONCLUDING REMARKS}

The C. elegans AC is an excellent model to investigate the various aspects underlying the complex process of cell invasion using an integrated approach by simultaneously examining: (1) cell fate acquisition, (2) establishment and maintenance of cell cycle arrest, (3) epigenetic and transcription factor networks that induce a pro-invasive gene expression pattern, (4) generation of extracellular cues that guide invading cells, (5) formation of invasive protrusions and finally (6) BM breaching. Since the $\mathrm{AC}$ does not migrate through the $\mathrm{BM}$ after breaching, it allows to separate cell invasion from later events occurring during cellular movements, thereby disentangling the different signaling pathways involved. Moreover, many transcription factors act in a context-dependent manner, underlining the importance of studying cell invasion in a physiological context. A remaining challenge is to understand the connections between the different layers controlling cell invasion, for example the link between G1 cell cycle arrest and the specification of the invasive fate. Many regulators of AC invasion are conserved and are associated with oncogenic processes in human cancer. Therefore, the unique AC of C. elegans could play a prominent role in solving the question of the proliferationinvasion interplay. 


\section{AUTHOR CONTRIBUTIONS}

EL wrote the first draft of the manuscript. All authors contributed to manuscript revision, read and approved the submitted version.

\section{REFERENCES}

Angel, P., Imagawa, M., Chiu, R., Stein, B., Imbra, R. J., Rahmsdorf, H. J., et al. (1987). Phorbol ester-inducible genes contain a common cis element recognized by a TPA-modulated trans-acting factor. Cell 49, 729-739. doi: 10.1016/0092-8674(87)90611-8

Attner, M. A., Keil, W., Benavidez, J. M., and Greenwald, I. (2019). HLH2/E2A expression links stochastic and deterministic elements of a cell fate decision during C. elegans gonadogenesis. Curr. Biol. 29, 3094-3100. doi: 10.1016/j.cub.2019.07.062

Banks, C. A. S., Miah, S., Adams, M. K., Eubanks, C. G., Thornton, J. L., Florens, L., et al. (2018). Differential HDAC1/2 network analysis reveals a role for prefoldin/CCT in HDAC1/2 complex assembly. Sci. Rep. 8:13712. doi: 10.1038/s41598-018-32009-w

Baonza, A., and Freeman, M. (2005). Control of cell proliferation in the drosophila eye by notch signaling. Dev. Cell 8, 529-539. doi: 10.1016/j.devcel.2005.01.019

Bard-Chapeau, E. A., Gunaratne, J., Kumar, P., Chua, B. Q., Muller, J., Bard, F. A., et al. (2013). EVI1 oncoprotein interacts with a large and complex network of proteins and integrates signals through protein phosphorylation. Proc. Natl. Acad. Sci. U.S.A. 110, 2885-2894. doi: 10.1073/pnas.1309310110

Bard-Chapeau, E. A., Jeyakani, J., Kok, C. H., Muller, J., Chua, B. Q., Gunaratne, J., et al. (2012). Ecotopic viral integration site 1 (EVI1) regulates multiple cellular processes important for cancer and is a synergistic partner for FOS protein in invasive tumors. Proc. Natl. Acad. Sci. U.S.A. 109, 2168-2173. doi: 10.1073/pnas.1119229109

Bayarmagnai, B., Perrin, L., Pourfarhangi, K. E., Graña, X., Tüzel, E., and Gligorijevic, B. (2019). Invadopodia-mediated ECM degradation is enhanced in the G1 phase of the cell cycle. J. Cell Sci. 132:jcs227116. doi: 10.1242/jcs.227116

Benbow, U., and Brinckerhoff, C. E. (1997). The AP-1 site and MMP gene regulation: what is all the fuss about? Matrix Biol. 15, 519-526. doi: 10.1016/S0945-053X(97)90026-3

Cáceres, R., Bojanala, N., Kelley, L. C., Dreier, J., Manzi, J., Federico, F. D., et al. (2018). Forces drive basement membrane invasion in Caenorhabditis elegans. Proc. Natl. Acad. Sci U.S.A. 115, 11537-11542. doi: 10.1073/pnas.1808760115

Campa, V. M., Gutiérrez-Lanza, R., Cerignoli, F., Díaz-Trelles, R., Nelson, B., Tsuji, T., et al. (2008). Notch activates cell cycle reentry and progression in quiescent cardiomyocytes. J. Cell Biol. 183, 129-141. doi: 10.1083/jcb.200806104

Chi, Y., Senyuk, V., Chakraborty, S., and Nucifora, G. (2003). EVI1 promotes cell proliferation by interacting with BRG1 and blocking the repression of BRG1 on E2F1 activity. J. Biol. Chem. 278, 49806-49811. doi: 10.1074/jbc.M3096 45200

Cohen, B., Shimizu, M., Izrailit, J., Ng, N. F. L., Buchman, Y., Pan, J. G., et al. (2010). Cyclin D1 is a direct target of JAG1-mediated Notch signaling in breast cancer. Breast Cancer Res. Treat. 123, 113-124. doi: 10.1007/s10549-009-0621-9

Dekker, C., Stirling, P. C., McCormack, E. A., Filmore, H., Paul, A., Brost, R. L., et al. (2008). The interaction network of the chaperonin CCT. EMBO J. 27, 1827-1839. doi: 10.1038/emboj.2008.108

Deng, T., Stempor, P., Appert, A., Daube, M., Ahringer, J., Hajnal, A., et al. (2020). The Caenorhabditis elegans homolog of the Evil protooncogene, egl-43, coordinates G1 cell cycle arrest with pro-invasive gene expression during anchor cell invasion. PLoS Genet. 16:e1008470. doi: 10.1371/journal.pgen.1008470

Gil-Henn, H., Patsialou, A., Wang, Y., Warren, M. S., Condeelis, J. S., and Koleske, A. J. (2013). Arg/Abl2 promotes invasion and attenuates proliferation of breast cancer in vivo. Oncogene 32, 2622-2630. doi: 10.1038/onc.2012.284

Greenwald, I., and Kovall, R. (2013). Notch signaling: genetics and structure. WormBook Online Rev. C Elegans Biol. 1-28. doi: 10.1895/wormbook.1.10.2

Haass, N. K., Beaumont, K. A., Hill, D. S., Anfosso, A., Mrass, P., Munoz, M. A., et al. (2014). Real-time cell cycle imaging during melanoma growth,

\section{FUNDING}

This work was supported by grants from the Swiss National Science Foundation (grant no. 31003A-166580) and the Swiss Cancer league (grant no. KFS-4377-02-2018).

invasion, and drug response. Pigment Cell Melanoma Res. 27, 764-776. doi: $10.1111 /$ pcmr.12274

Hagedorn, E. J., Yashiro, H., Ziel, J. W., Ihara, S., Wang, Z., and Sherwood, D. R. (2009). Integrin acts upstream of netrin signaling to regulate formation of the anchor cell's invasive membrane in C. elegans. Dev. Cell 17, 187-198. doi: 10.1016/j.devcel.2009.06.006

Hatzikirou, H., Basanta, D., Simon, M., Schaller, K., and Deutsch, A. (2012). 'Go or Grow': the key to the emergence of invasion in tumour progression? Math. Med. Biol. J. IMA 29, 49-65. doi: 10.1093/imammb/dqq011

Hoek, K. S., Eichhoff, O. M., Schlegel, N. C., Döbbeling, U., Kobert, N., Schaerer, L., et al. (2008). In vivo switching of human melanoma cells between proliferative and invasive states. Cancer Res. 68, 650-656. doi: 10.1158/0008-5472.CAN-07-2491

Hwang, B. J., Meruelo, A. D., and Sternberg, P. W. (2007). C. elegans EVI1 protooncogene, EGL-43, is necessary for Notch-mediated cell fate specification and regulates cell invasion. Development 134, 669-679. doi: 10.1242/dev.02769

Hwang, B. J., and Sternberg, P. W. (2004). A cell-specific enhancer that specifies lin-3 expression in the $C$. elegans anchor cell for vulval development. Development 131, 143-151. doi: 10.1242/dev.00924

Ihara, S., Hagedorn, E. J., Morrissey, M. A., Chi, Q., Motegi, F., Kramer, J. M., et al. (2011). Basement membrane sliding and targeted adhesion remodels tissue boundaries during uterine-vulval attachment in C. elegans. Nat. Cell Biol. 13, 641-651. doi: $10.1038 /$ ncb2233

Ivanochko, D., Halabelian, L., Henderson, E., Savitsky, P., Jain, H., Marcon, E., et al. (2019). Direct interaction between the PRDM3 and PRDM16 tumor suppressors and the NuRD chromatin remodeling complex. Nucleic Acids Res. 47, 1225-1238. doi: 10.1093/nar/gky1192

Izutsu, K., Kurokawa, M., Imai, Y., Maki, K., Mitani, K., and Hirai, H. (2001). The corepressor CtBP interacts with Evi-1 to repress transforming growth factor $\beta$ signaling. Blood 97, 2815-2822. doi: 10.1182/blood.V97.9.2815

Karp, X., and Greenwald, I. (2004). Multiple roles for the E/Daughterless ortholog HLH-2 during C. elegans gonadogenesis. Dev. Biol. 272, 460-469. doi: 10.1016/j.ydbio.2004.05.015

Kelley, L. C., Chi, Q., Cáceres, R., Hastie, E., Schindler, A. J., Jiang, Y., et al. (2019). Adaptive F-Actin Polymerization and Localized ATP Production Drive Basement Membrane Invasion in the Absence of MMPs. Dev. Cell 48, 313-328. doi: 10.1016/j.devcel.2018.12.018

Kimble, J. (1981). Alterations in cell lineage following laser ablation of cells in the somatic gonad of Caenorhabditis elegans. Dev. Biol. 87, 286-300. doi: 10.1016/0012-1606(81)90152-4

Kimble, J., and Hirsh, D. (1979). The postembryonic cell lineages of the hermaphrodite and male gonads in Caenorhabditis elegans. Dev. Biol. 70, 396-417. doi: 10.1016/0012-1606(79)90035-6

Kohrman, A. Q., and Matus, D. Q. (2017). Divide or conquer: cell cycle regulation of invasive behavior. Trends Cell Biol. 27, 12-25. doi: 10.1016/j.tcb.2016.08.003

Lee, W., Mitchell, P., and Tjian, R. (1987). Purified transcription factor AP-1 interacts with TPA-inducible enhancer elements. Cell 49, 741-752. doi: 10.1016/0092-8674(87)90612-X

Lohmer, L. L., Clay, M. R., Naegeli, K. M., Chi, Q., Ziel, J. W., Hagedorn, E. J., et al. (2016). A sensitized screen for genes promoting invadopodia function in vivo: CDC-42 and Rab GDI-1 direct distinct aspects of invadopodia formation. PLoS Genet 12:e1005786. doi: 10.1371/journal.pgen.1005786

Matus, D. Q., Li, X.-Y., Durbin, S., Agarwal, D., Chi, Q., Weiss, S. J., et al. (2010). In vivo identification of regulators of cell invasion across basement membranes. Sci. Signal 3:ra35. doi: 10.1126/scisignal.2000654

Matus, D. Q., Lohmer, L. L., Kelley, L. C., Schindler, A. J., Kohrman, A. Q., Barkoulas, M., et al. (2015). Invasive cell fate requires G1 cell-cycle arrest and histone deacetylase-mediated changes in gene expression. Dev. Cell 35, 162-174. doi: 10.1016/j.devcel.2015.10.002 
Medwig-Kinney, T. N., Smith, J. J., Palmisano, N. J., Tank, S., Zhang, W., and Matus, D. Q. (2020). A developmental gene regulatory network for $C$. elegans anchor cell invasion. Development 147:dev185850. doi: 10.1242/dev.1 85850

Naegeli, K. M., Hastie, E., Garde, A., Wang, Z., Keeley, D. P., Gordon, K. L., et al. (2017). Cell invasion in vivo via rapid exocytosis of a transient lysosome-derived membrane domain. Dev. Cell 43, 403-417. doi: 10.1016/j.devcel.2017.10.024

Noseda, M., Niessen, K., McLean, G., Chang, L., and Karsan, A. (2005). Notch-dependent cell cycle arrest is associated with downregulation of minichromosome maintenance proteins. Circ. Res. 97, 102-104. doi: 10.1161/01.RES.0000174380.06673.81

Ozanne, B. W., Spence, H. J., McGarry, L. C., and Hennigan, R. F. (2006). Transcription factors control invasion: AP-1 the first among equals. Oncogene 26, 1-10. doi: 10.1038/sj.onc.1209759

Patsialou, A., Wang, Y., Pignatelli, J., Chen, X., Entenberg, D., Oktay, M., et al. (2015). Autocrine CSF1R signaling mediates switching between invasion and proliferation downstream of TGF $\beta$ in claudin-low breast tumor cells. Oncogene 34, 2721-2731. doi: 10.1038/onc.2014.226

Qian, X., Hulit, J., Suyama, K., Eugenin, E. A., Belbin, T. J., Loudig, O., et al. (2013). p21CIP1 mediates reciprocal switching between proliferation and invasion during metastasis. Oncogene 32, 2292-2303. doi: 10.1038/onc.2012.249

Ranganathan, P., Vasquez-Del Carpio, R., Kaplan, F. M., Wang, H., Gupta, A., VanWye, J. D., et al. (2011). Hierarchical phosphorylation within the ankyrin repeat domain defines a phosphoregulatory loop that regulates notch transcriptional activity. J. Biol. Chem. 286, 28844-28857. doi: $10.1074 /$ jbc.M111.243600

Rimann, I., and Hajnal, A. (2007). Regulation of anchor cell invasion and uterine cell fates by the egl-43 Evi-1 proto-oncogene in Caenorhabditis elegans. Dev. Biol. 308, 187-195. doi: 10.1016/j.ydbio.2007.05.023

Ronchini, C., and Capobianco, A. J. (2001). Induction of cyclin D1 transcription and CDK2 activity by notchic: implication for cell cycle disruption in transformation by notchic. Mol. Cell. Biol. 21, 5925-5934. doi: 10.1128/MCB.21.17.5925-5934.2001

Sallee, M. D., Littleford, H. E., and Greenwald, I. (2017). A bHLH code for sexually dimorphic form and function of the C. elegans somatic gonad. Curr. Biol. 27, 1853-1860. doi: 10.1016/j.cub.2017.05.059

Sarmento, L. M., Huang, H., Limon, A., Gordon, W., Fernandes, J., Tavares, M. J., et al. (2005). Notch1 modulates timing of G1-S progression by inducing SKP2 transcription and p27Kip1 degradation. J. Exp. Med. 202, 157-168. doi: $10.1084 /$ jem.20050559

Seydoux, G., and Greenwald, I. (1989). Cell autonomy of lin-12 function in a cell fate decision in C. elegans. Cell 57, 1237-1245. doi: 10.1016/0092-8674(89)90060-3
Sherwood, D. R., Butler, J. A., Kramer, J. M., and Sternberg, P. W. (2005). FOS1 promotes basement-membrane removal during anchor-cell invasion in $C$. elegans. Cell 121, 951-962. doi: 10.1016/j.cell.2005.03.031

Sherwood, D. R., and Sternberg, P. W. (2003). Anchor cell invasion into the vulval epithelium in C. elegans. Dev. Cell 5, 21-31. doi: 10.1016/S1534-5807(03)00168-0

Sternberg, P. W. (2005). Vulval development. WormBook Online Rev. C Elegans Biol. 1-28. doi: 10.1895/wormbook.1.6.1

Unhavaithaya, Y., Shin, T. H., Miliaras, N., Lee, J., Oyama, T., and Mello, C. C. (2002). MEP-1 and a homolog of the NURD complex component Mi-2 act together to maintain germline-soma distinctions in C. elegans. Cell 111, 991-1002. doi: 10.1016/S0092-8674(02)01202-3

Wang, L., Shen, W., Lei, S., Matus, D., Sherwood, D., and Wang, Z. (2014). MIG-10 (Lamellipodin) stabilizes invading cell adhesion to basement membrane and is a negative transcriptional target of EGL-43 in C. elegans. Biochem. Biophys. Res. Commun. 452, 328-333. doi: 10.1016/j.bbrc.2014.08.049

Wang, Z., Chi, Q., and Sherwood, D. R. (2014a). MIG-10 (lamellipodin) has netrin-independent functions and is a FOS-1A transcriptional target during anchor cell invasion in C. elegans. Dev. Camb. Engl. 141, 1342-1353. doi: $10.1242 / \mathrm{dev} .102434$

Wang, Z., Linden, L. M., Naegeli, K. M., Ziel, J. W., Chi, Q., Hagedorn, E. J., et al. (2014b). UNC-6 (netrin) stabilizes oscillatory clustering of the UNC-40 (DCC) receptor to orient polarity. J. Cell Biol. 206, 619-633. doi: $10.1083 /$ jcb.201405026

Yoshimi, A., Goyama, S., Watanabe-Okochi, N., Yoshiki, Y., Nannya, Y., Nitta, E., et al. (2011). Evil represses PTEN expression and activates PI3K/AKT/mTOR via interactions with polycomb proteins. Blood 117, 3617-3628. doi: 10.1182/blood-2009-12-261602

Ziel, J. W., Hagedorn, E. J., Audhya, A., and Sherwood, D. R. (2009). UNC-6 (netrin) orients the invasive membrane of the anchor cell in C. elegans. Nat. Cell Biol. 11, 183-189. doi: 10.1038/ncb1825

Conflict of Interest: The authors declare that the research was conducted in the absence of any commercial or financial relationships that could be construed as a potential conflict of interest.

Copyright (๑) 2021 Lattmann, Deng and Hajnal. This is an open-access article distributed under the terms of the Creative Commons Attribution License (CC BY).

The use, distribution or reproduction in other forums is permitted, provided the original author(s) and the copyright owner(s) are credited and that the original publication in this journal is cited, in accordance with accepted academic practice. No use, distribution or reproduction is permitted which does not comply with these terms. 